\title{
SEM and TEM Study of a Ceramic Membrane/Laser Induced Graphene Composite
}

\author{
M. Bayati ${ }^{1}$, H. Peng ${ }^{1}$, M. Fidalgo de Cortalezzi ${ }^{1}$, H. Deng ${ }^{2}$, Jian Lin $^{2}$ and T. A. White ${ }^{3}$ \\ 1. Civil and Environmental Engineering, University of Missouri, MO, USA \\ 2. Mechanical \& Aerospace Engineering, University of Missouri, MO, USA \\ ${ }^{3 .}$ Electron Microscopy Core Facility, University of Missouri, MO, USA
}

Graphene has attracted a lot of attention in diverse branches of science and technology since first reported in 2004 [1]. With its persistent properties, graphene has displayed great potential for atomic permeation, water transport, and gas separation [2]. In this study, we report a novel technique for one-step fabrication of a uniform, stable, and strongly bonded graphene layer on a microporous ceramic membrane acting as support material. A composite ceramic-laser induced graphene (LIG) membrane was fabricated using one step scalable way, as described by Lin et al [3]. A titanium dioxide ceramic support (pore size $1.4 \mu \mathrm{m}$, diameter $47 \mathrm{~mm}$, Sterlitech) was spin-coated with a $12.0 \mathrm{wt} \%$ solution of poly (pyromellitic dianhydrideco-4,4'-oxidianiline, amic acid) (PAA), which was then transformed to polyimide (PI) by thermal imidization. The PI was then irradiated using $\mathrm{H}$-series Desktop $\mathrm{CO}_{2}$ laser to photothermally convert the $\mathrm{sp}^{3}$-carbon atoms to $\mathrm{sp}^{2}$-carbon atoms. The laser was operated at $10 \mathrm{~W}$ power, 24 inch.s ${ }^{-1}$ raster speed, and 1000X1000 dpi raster mode.

FEI Quanta 600 FEG Environmental Scanning Electron Microscopy (ESEM) was used to capture high resolution images for the membrane surface and cross section. SEM images were collected using accelerating voltage $5 \mathrm{kV}$, working distance $10 \mathrm{~mm}$, aperture 6 , spot size 3, and Everhart-Thornley Detector (ETD) was used to detect secondary electron (SE). For the cross-section preparation, an IsoMet low speed saw with diamond blade have been used to cut the samples. Then, the samples were polished using diamond lapping films $9 \mu \mathrm{m}, 3 \mu \mathrm{m}$, and $1 \mu \mathrm{m}$, consecutively. Figure 1(a,b) show that the 4 layers of PI were totally converted to graphene. The PI thickness on the top of ceramic membrane was between 1618 um (figure $2 \mathrm{a}$ ), and the thickness of LIG after using the laser was between $40 \mu \mathrm{m}$ and $43 \mu \mathrm{m}$ (Figure 2 b). FEI Tecnai F30 twin Transmission Electron Microscopy (TEM) was used to capture high resolution images for LIG flakes. Figure $3(\mathrm{~b}, \mathrm{c})$ show the TEM images which were collected at $100 \mathrm{kV}$ using aperture 4 for C2. The X-Ray Diffraction (XRD) analysis (Figure 3 a) showed an intense peak at $2 \theta=25.9^{\circ}$, which gives an interlayer spacing of $3.4 \AA$ between (002) planes in the LIG, indicating a high degree of graphitization. This observation is in good agreement with the TEM images (Figure $3 \mathrm{c}$ ). Another peak at $2 \theta=42.9^{\circ}$ indicates (100) reflections which are associated with an in-plane structure.

The new composite membrane is a promising new material for membrane distillation or electro osmosis, where the hydrophobicity and electrical conductivity of the graphene layer may provide important advantages over current membrane materials. 
References:

[1] K.S Novoselov et al, Science 306 (2004), p. 666.

[2] Tsetseris, L. and S.T. Pantelides, Carbon 67 (2014), p. 58.

[3] J Lin et al, Nat Commun 5 (2014), p. 5714.
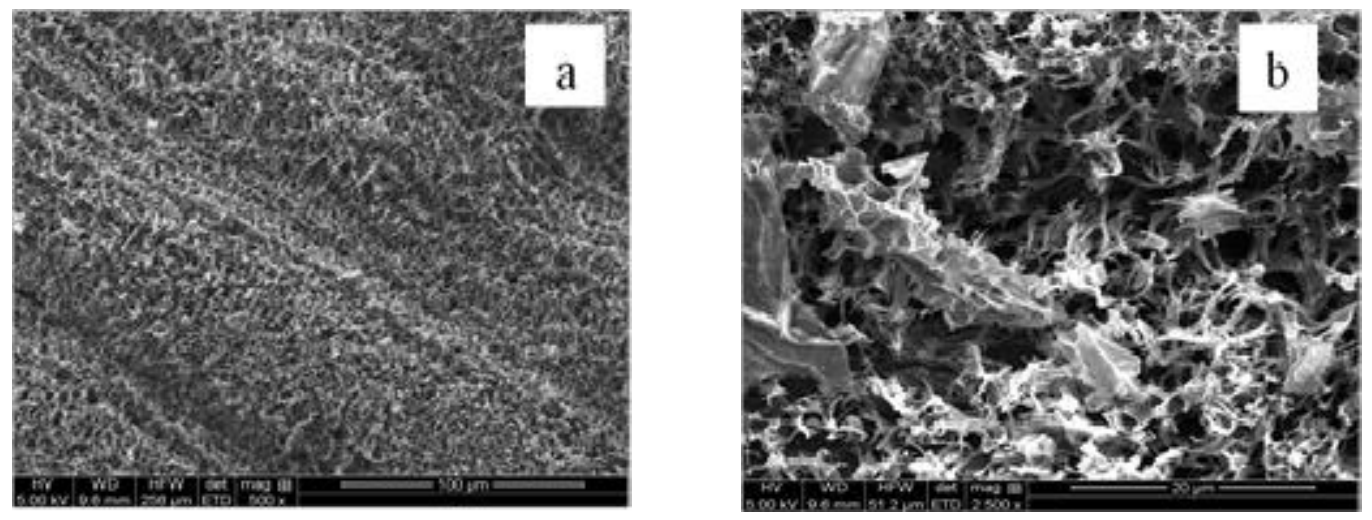

Figure 1. SEM images for LIG on ceramic membrane. (a) LIG after using laser with 500x mag. (b) LIG with 2500x mag.
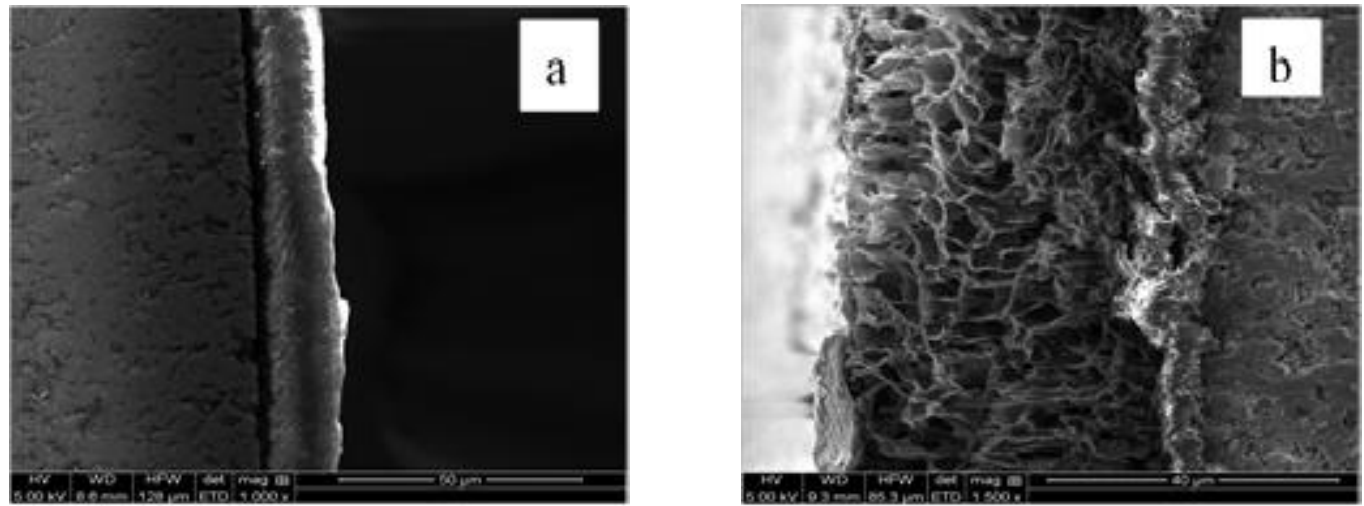

Figure 2. SEM cross-section images. (a) 4-layer coating PI with thickness 16-18 um. (b) LIG with thickness 40-43 um.
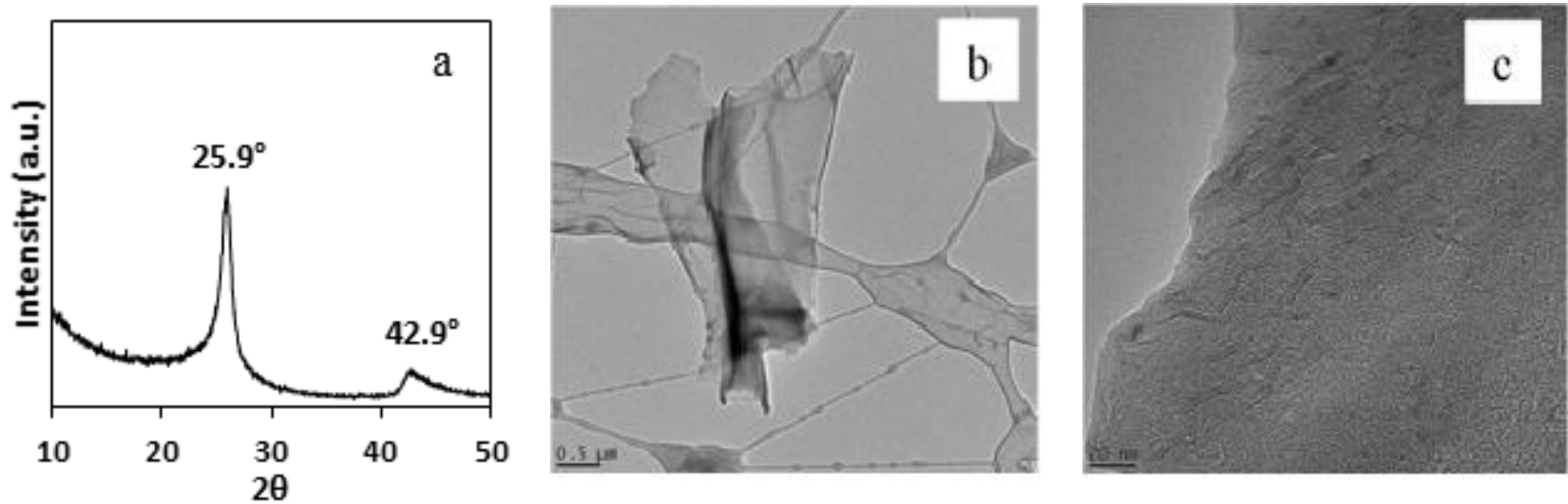

Figure 3. (a) X-ray diffraction patterns of LIG. (b) TEM image of LIG flakes. (c) LIG Average lattice space of $3.4 \AA$ corresponds to the (002) planes of graphitic materials. 